\title{
Electroacupuncture effects on the P2X4R pathway in microglia regulating the excitability of neurons in the substantia gelatinosa region of rats with spinal nerve ligation
}

\author{
YUYIN ZHENG ${ }^{1,2^{*}}$, CHENGQIAN JIA ${ }^{1-3^{*}}$, XIA JIANG $^{1,2}$, JIE CHEN $^{1,2}$, XIAO-LONG CHEN $^{1,2}$, \\ XINWANG YING ${ }^{1,2}$, JIAYU WU ${ }^{1,2}$, MINGCHEN JIANG ${ }^{2,4}$, GUANHU YANG ${ }^{1,2}$, \\ WENZHAN TU ${ }^{1,2}$, KECHENG ZHOU $^{1,2}$ and SONGHE JIANG ${ }^{1,2}$ \\ ${ }^{1}$ Department of Physical Medicine and Rehabilitation, The Second Affiliated Hospital and Yuying Children's \\ Hospital of Wenzhou Medical University; ${ }^{2}$ China-USA Institute for Acupuncture and Rehabilitation, \\ Integrative and Optimized Medicine Research Center, Wenzhou Medical University, Wenzhou, Zhejiang 325027; \\ ${ }^{3}$ Department of Pain, The Central Hospital of Wuhan, Tongji Medical College, Huazhong University \\ of Science and Technology, Wuhan, Hubei 430014, P.R. China; ${ }^{4}$ Department of Physiology, \\ Northwestern University Feinberg School of Medicine, Chicago, IL 60208, USA
}

Received April 24, 2019; Accepted May 5, 2020

DOI: $10.3892 / \mathrm{mmr} .2020 .11814$

\begin{abstract}
Electroacupuncture (EA) has been used to treat neuropathic pain induced by peripheral nerve injury (PNI) by applying an electrical current to acupoints with acupuncture needles. However, the mechanisms by which EA treats pain remain indistinct. High $\mathrm{P} 2 \mathrm{X} 4$ receptor (P2X4R) expression levels demonstrate a notable increase in hyperactive microglia in the ipsilateral spinal dorsal horn following PNI. In order to demonstrate the possibility that EA analgesia is mediated in part by $\mathrm{P} 2 \mathrm{X} 4 \mathrm{R}$ in hyperactive microglia, the present study performed mechanical withdrawal threshold (MWT) and thermal withdrawal latency (TWL) tests in male Sprague-Dawley rats that had undergone spinal nerve ligation (SNL). The expression levels of spinal P2X4R were determined using reverse transcription-quantitative PCR, western blotting analysis and immunofluorescence staining. Furthermore, spontaneous excitatory postsynaptic currents (sEPSCs) were recorded using whole-cell patch clamp to demonstrate the effect of EA on synaptic transmission in rat
\end{abstract}

Correspondence to: Professor Songhe Jiang or Dr Kecheng Zhou, Department of Physical Medicine and Rehabilitation, The Second Affiliated Hospital and Yuying Children's Hospital of Wenzhou Medical University, 268 Xueyuanxi Road, Wenzhou, Zhejiang 325027, P.R. China

E-mail: songhe.jiang@gmail.com

E-mail: zhou_kc@126.com

${ }^{*}$ Contributed equally

Key words: electroacupuncture, $\mathrm{P} 2 \mathrm{X} 4$ receptor, microglia, neuropathic pain spinal substantia gelatinosa (SG) neurons. The results of the present study demonstrated that EA increased the MWT and TWL and decreased overexpression of P2X4R in hyperactive microglia in SNL rats. Moreover, EA attenuated the frequency of sEPSCs in SG neurons in SNL rats. The results of the present study indicate that EA may mediate P2X4R in hyperactive spinal microglia to inhibit nociceptive transmission of SG neurons, thus relieving pain in SNL rats.

\section{Introduction}

Electroacupuncture (EA) is used to treat neuropathic pain (NP) induced by peripheral nerve injury (PNI) $(1,2)$. To the best of our knowledge, however, the mechanism has not yet been identified. Previous studies have noted that neuronal activities are associated with neurotransmitters and neuromodulators, such as opioids, interleukins, chemokines, serotonin and adenosine, and that the appearance and persistence of NP depends on hyperactive microglia (3-8). Suppression of microglia activation attenuates pain induced by nerve injury $(9,10)$. Previous studies have demonstrated that the $\mathrm{P} 2 \mathrm{X} 4$ receptor (P2X4R) triggers allodynia following PNI, and that relief of NP occurs both in mice injected intrathecally with a P2X4R antisense oligonucleotide and in mice lacking P2X4R $(8,11)$. ATP is a transmitter that conveys sensory information between hyperactive microglia and nociceptive neurons. Hyperactive microglia induce or promote increased expression levels of P2X4R (8). In response to extracellular ATP, P2X4R can mediate a number of effects, such as the production and diffusion of bioactive factors, including cytokines and neurotrophic factors that can induce depolarization of dorsal horn sensory neurons (12). The physiological process of EA analgesia involves numerous transmitters and modulators, including acetylcholine opioid peptides, substance $\mathrm{P}$, glutamate, $\gamma$-amino-butyric acid and other associated peptides (13). Previous studies have indicated 
that EA analgesia is associated with decreased purine and purinergic receptors, especially P2X4R $(3,14)$. In light of this association, EA treatment may have a role in the relief of NP, which may be mediated in part by $\mathrm{P} 2 \mathrm{X} 4 \mathrm{R}$ in microglia in the spinal dorsal horn (SDH) $(15,16)$. The present study demonstrated that the pain behavior and expression levels of P2X4R in microglia in the SDH were altered in spinal nerve ligation (SNL) rats. Whole-cell patch clamp techniques were used to investigate the variation in the frequency of spontaneous excitatory postsynaptic currents (sEPSC) in SNL rat spinal substantia gelatinosa (SG) neurons. Studies have demonstrated that EA treatment at 'Zusanli' (ST-36, at the posterolateral aspect of the knee joint, $\sim 5 \mathrm{~mm}$ below the humeral head) ) and 'Kunlun' (BL-60, 10 mm above the prominence of the lateral malleolus of the hind limb) points can relieve neuropathic pain, but its specific mechanism has not yet been elucidated $(1,2)$. The aim of the present study was to investigate the potential mechanism by which EA treatment at 'Zusanli' and 'Kunlun' points relieves NP via the action on P2X4R.

\section{Materials and methods}

Experimental animals. The Institutional Animal Care and Use Committee of Wenzhou Medical University approved all experiments. A total of 72 male Sprague-Dawley rats (weight, 180-200 g; age, 6-8 weeks), bought from Wenzhou Medical University (Wenzhou, China), were kept on a standard laboratory diet at room temperature $\left(20-22^{\circ} \mathrm{C}\right)$ and 12 -h alternative light-dark cycle conditions in a pathogen-free room. The behavioral experiment was performed between 2:00 p.m. and 4:00 p.m. All experimental rats were randomly distributed into four groups $(n=6)$ : Control, sham, SNL and ipsilateral EA groups. All surgical procedures were performed using a microscope (Leica S8 APO; Leica Microsystems, Ltd; magnification, $\mathrm{x} 4)$. The experimental rats were anesthetized with $5 \%$ chloral hydrate $[350 \mathrm{mg} / \mathrm{kg}$, intraperitoneal (i.p.)]. SNL surgery was performed as previously described (17-19). Briefly, an incision was made in the midline lumbar region of animals placed in a prone position. In order to expose the right L4-L5 spinal nerves completely, the right L5 vertebral transverse was cut. Following right L5 spinal nerve separation, it was ligated with 5-0 silk, and the incision was closed. In the sham group, the right L5 spinal nerve was exposed but not ligated. Pain thresholds were measured at days $0,3,5,7,10,12$ and 14 post-SNL.

Behavioral tests. The EA treatment time was fixed at 9:00-10:00 a.m. MWT and TWL tests time were fixed at 2:00-4:00 p.m. The Electronic von Frey anesthesiometer (IITC Life Science Inc.) was used to measure MWT to judge mechanical hyperalgesia. The experimental rats were allowed to acclimatize in the wire mesh-bottom cages $(20 \times 14 \times 16 \mathrm{~cm})$ for $30 \mathrm{~min}$. The test probe was positioned at the base of third and fourth toes, and the pressure of the Electronic von Frey anesthesiometer was set to 0.1-70.0 g. Both lifting and licking the paw were considered to be positive responses. The maximum pressure was also recorded. Each hind paw was tested alternately six times at 5 min intervals. The average value was used for statistical analysis. Following the MWT test, rats were placed in a square, transparent, bottomless acrylic box $(16.0 \times 12.5 \times 14.0 \mathrm{~cm})$ and allowed to acclimatize for 15 min before being subjected to a TWL test. The TWL was used to test thermal hyperalgesia using Plantar Test apparatus (Ugo-Basile S.R.L.) (13). The infrared source was set at $60^{\circ} \mathrm{C}$ under a glass plate and directed towards the plantar surface of the hind paw. Withdrawal of the paw led to the infrared source breaking off, at which point latency was measured. The hind paw of the experimental rats was tested five times at 15 -min intervals and the TWL was expressed as the mean value.

EA treatment. EA treatment was started on day 7 post-SNL in the EA groups. The rats were maintained in fixation equipment (patent no. 201110021482.5; State Intellectual Property Office) (13). Acupuncture needles were percutaneously inserted 2-3 $\mathrm{mm}$ at the Zusanli and Kunlun points. EA stimulus $(2 / 100 \mathrm{~Hz} ; 1.5 \mathrm{~mA})$ was delivered using an electrical stimulation device (HANS-200E; Nanjing Jisheng Medical Technology, Ltd.) for $30 \mathrm{~min}$ daily. The intensity was set at $1 \mathrm{~mA}$, and the total stimulation period was $30 \mathrm{~min}$ for 7 days, which ensured the best curative effect of EA (Fig. 1).

Reverse transcription-quantitative PCR. Real-time amplification using SYBR-Green Supermix (Toyobo Life Science) and a Light Cycler 480 system (Roche Diagnostics $\mathrm{GmbH}$ ) was performed using $4 \mathrm{ng}$ cDNA extracted from L4-L5 segments with TRIzol ${ }^{\circledR}$ (Invitrogen; Thermo Fisher Scientific, Inc.) according to the manufacturer's instructions. RNA preparation and cDNA synthesis were performed as previously described (20). The PCR conditions consisted of an initial melting cycle at $95^{\circ} \mathrm{C}$ for $15 \mathrm{~min}$, followed by 40 cycles of amplification at $95^{\circ} \mathrm{C}$ for $15 \mathrm{sec}$ (denaturation), $60^{\circ} \mathrm{C}$ for $30 \mathrm{sec}$ (annealing) and $72^{\circ} \mathrm{C}$ for $30 \mathrm{sec}$ (extension). Primers were procured from Invitrogen (Thermo Fisher Scientific, Inc.): P2X4R forward, 5'-GGGTGAAGTTTTATTCCAGC-3'; P2X4R reverse, 5'-GGGTGAAGTTTTCTGCAGCC-3'; GAPDH forward, 5'-CTTCACCACCATGGAGAAGGC-3'; and GAPDH reverse, 5'-GGCATGGACTGTGGTCATGAG-3'. The quantification values were obtained from the quantification cycle $(\mathrm{Cq})$ number at which the increase in the signal was associated with exponential growth of the PCR products. All samples were run in triplicate and repeated three times. RPS16 quantification was used as an internal control for normalization. Fold differences in mRNA levels over vehicle control were calculated using the $2^{-\Delta \Delta \mathrm{Cq}}$ method (21).

Western blotting. Western blotting was performed as previously described (22) with minor modifications. The rats were deeply anesthetized with i.p. injection of $30 \mathrm{mg} / \mathrm{kg}$ pentobarbital sodium. The Rat Anesthesia Guidelines of University Minnesota (https://www.researchservices.umn.edu/ services-name/research-animal-resources/research-support/ guidelines/anesthesia-rats) were used to determine when the rat had entered deep anesthesia. Corneal reflexes were observed to disappear in the rat eye. Rats were observed to have no response after lightly clamping the fourth toe with tweezers, thereby confirming that rats had entered a state of deep anesthesia. The rats were sacrificed by decapitation. The proteins extracted from L4-L5 segments were quantified using a bicinchoninic acid protein assay kit (Beyotime Institute of Biotechnology). The proteins (42 kDa actin and 


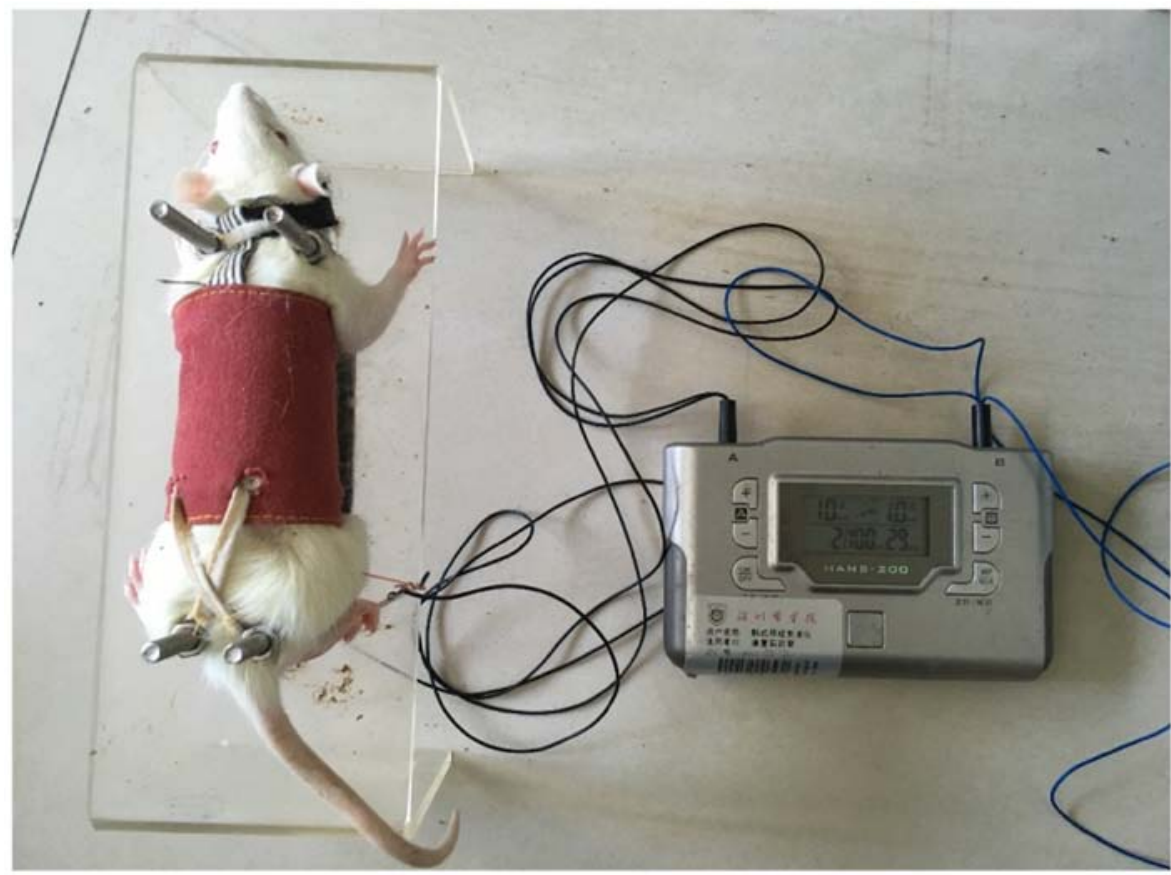

Figure 1. Use of comfortable fixation equipment can decrease stress and enhance convenience in acupuncture research.

$62 \mathrm{kDa}$ P2X4R) were subjected to $8 \%$ SDS-PAGE and transferred to a PVDF membrane. The membranes were blocked with $5 \%$ non-fat milk for $2 \mathrm{~h}$ at $4^{\circ} \mathrm{C}$ and incubated overnight at $4^{\circ} \mathrm{C}$ with anti-rat $\mathrm{P} 2 \mathrm{X} 4 \mathrm{R}$ polyclonal antibody $(1: 1,000$; Alomone Labs) or GAPDH antibody (1:4,000; Sigma-Aldrich, Merck). Membranes were subsequently incubated with horseradish peroxidase (HRP)-conjugated secondary antibody (goat anti-rabbit IgG; 1:3,000; cat. no. 7074; Cell Signaling Technology, Inc.) for $2 \mathrm{~h}$ at $4^{\circ} \mathrm{C}$. These membranes were washed 3 times with TBST $(0.1 \%$ Tween-20) $(5 \mathrm{~min} /$ time $)$ after incubating with HRP-conjugated antibody. The bands were detected using the ECL method (BeyoECL Plus; cat. no. P0018S; Beyotime Institute of Biotechnology) and exposed to radiography films.

Immunofluorescence staining. Immunofluorescence staining was performed as previously described with minor modifications (23). On day 14 post-SNL, the rats were anesthetized with $10 \%$ chloral hydrate $(350 \mathrm{mg}$ chloral hydrate $/ \mathrm{kg}$ ). The rats exhibited no signs of peritonitis following administration of chloral hydrate. The rats were perfused through the ascending aorta with physiological saline. Subsequently, rats were fixed with $4 \%$ paraformaldehyde for $4 \mathrm{~h}$ in $0.1 \mathrm{M}$ phosphate buffer at $\mathrm{pH}$ 7.2-7.4, $4^{\circ} \mathrm{C}$. Following fixation, the heartbeat disappeared and the body was stiff. The lumbosacral section was dehydrated, cleared and embedded in paraffin for transverse paraffin sections. Transverse spinal cord sections $(5 \mu \mathrm{m})$ were excised and mounted on poly-L-lysine-coated slides. Sections were deparaffinized and rehydrated in descending alcohol series. Then, sections were immersed in antigen repair buffer (sodium citrate; $\mathrm{pH}$ 6.0) and heated in a microwave oven at $100^{\circ} \mathrm{C}$ for $20 \mathrm{~min}$ and allowed to cool naturally. The slides were blocked with $3 \% \mathrm{H}_{2} \mathrm{O}_{2}$ for $10 \mathrm{~min}$ at room temperature and $10 \%$ normal goat serum (Gibco; Thermo Fisher Scientific, Inc.) with $0.3 \%$ Triton X-100 in PBS for $1 \mathrm{~h}$ at $4^{\circ} \mathrm{C}$.
The sections were incubated with rabbit anti-P2X4 (1:200; cat. no. 13534-1-AP; ProteinTech Group Inc.) and mouse anti-ionized calcium-binding adapter molecule 1 (Iba-1; 1:400; cat. no. ab15690; Abcam) antibodies for $16 \mathrm{~h}$ at $4^{\circ} \mathrm{C}$. The secondary antibodies were tetraethyl rhodamine isothiocyanate (1:1,000; cat. no. AP31444TC-N; OriGene Technologies, Inc.) conjugated to rabbit anti-P2X4 IgG and fluorescein conjugated to goat anti-mouse IgG (1:5,000; cat. no. BL003A; Biosharp Life Sciences), and the incubation was performed for $1 \mathrm{~h}$ at $37^{\circ} \mathrm{C}$. Slides were washed three times $(5 \mathrm{~min} / \mathrm{time})$ with PBS and incubated with DAPI staining solution $(1: 1,000$; cat. no. C1005; Beyotime Institute of Biotechnology) for 10 min at $25^{\circ} \mathrm{C}$, then washed a further three times with PBS (5 min/time). Images were captured using a BX41 fluorescence microscope (Olympus Corporation; magnification, x10 and $\mathrm{x} 40$ ). Image-Pro Plus software (version 5.1; Media Cybernetics, Inc.) was used to determine the staining intensity.

Section preparation. The spinal cord sections from rats were prepared as previously described $(13,24)$. Briefly, rats were anesthetized as aforementioned, then transcardially perfused with $\sim 70 \mathrm{ml}$ of ice-cold, oxygenated $\left(95 \% \mathrm{O}_{2}, 5 \% \mathrm{CO}_{2}\right)$ cutting solution containing: $105.0 \mathrm{~N}$-methyl-D-glucamine, 105.0 $\mathrm{HCl}$, 2.5.0 KCl, 1.2 $\mathrm{NaH}_{2} \mathrm{PO}_{4}, 26.0 \mathrm{NaHCO}_{3}, 25.0$ glucose, $10.0 \mathrm{MgSO}_{4}, 0.5 \mathrm{CaCl}_{2}, 5.0 \mathrm{~L}$-ascorbic acid, 3.0 sodium pyruvate and $2.0 \mathrm{mM}$ thiourea ( $\mathrm{pH} 7.4,295-305 \mathrm{mOsm})$. The lumbosacral section was removed in the cutting solution. All ventral and dorsal roots were cut and the pia-arachnoid membrane was removed. Transverse spinal sections $(300 \mu \mathrm{m})$ were cut using a vibratome (VT1200S; Leica Microsystems, Ltd.) and placed in an incubator filled with normal oxygenated $\left(95 \% \mathrm{O}_{2}, 5 \% \mathrm{CO}_{2}\right) \mathrm{Krebs}$ solution for at least $30 \mathrm{~min}$ at $32^{\circ} \mathrm{C}$. The normal Krebs solution contained: $117.0 \mathrm{NaCl}$, 3.6 KCl, 2.5 $\mathrm{CaCl}_{2}, 1.2 \mathrm{MgCl}_{2}, 1.2 \mathrm{NaH}_{2} \mathrm{PO}_{4}, 25.0 \mathrm{NaHCO}_{3}$, $11.0 \mathrm{D}$-glucose, 0.4 ascorbic acid and $2.0 \mathrm{mM}$ pyruvate. 

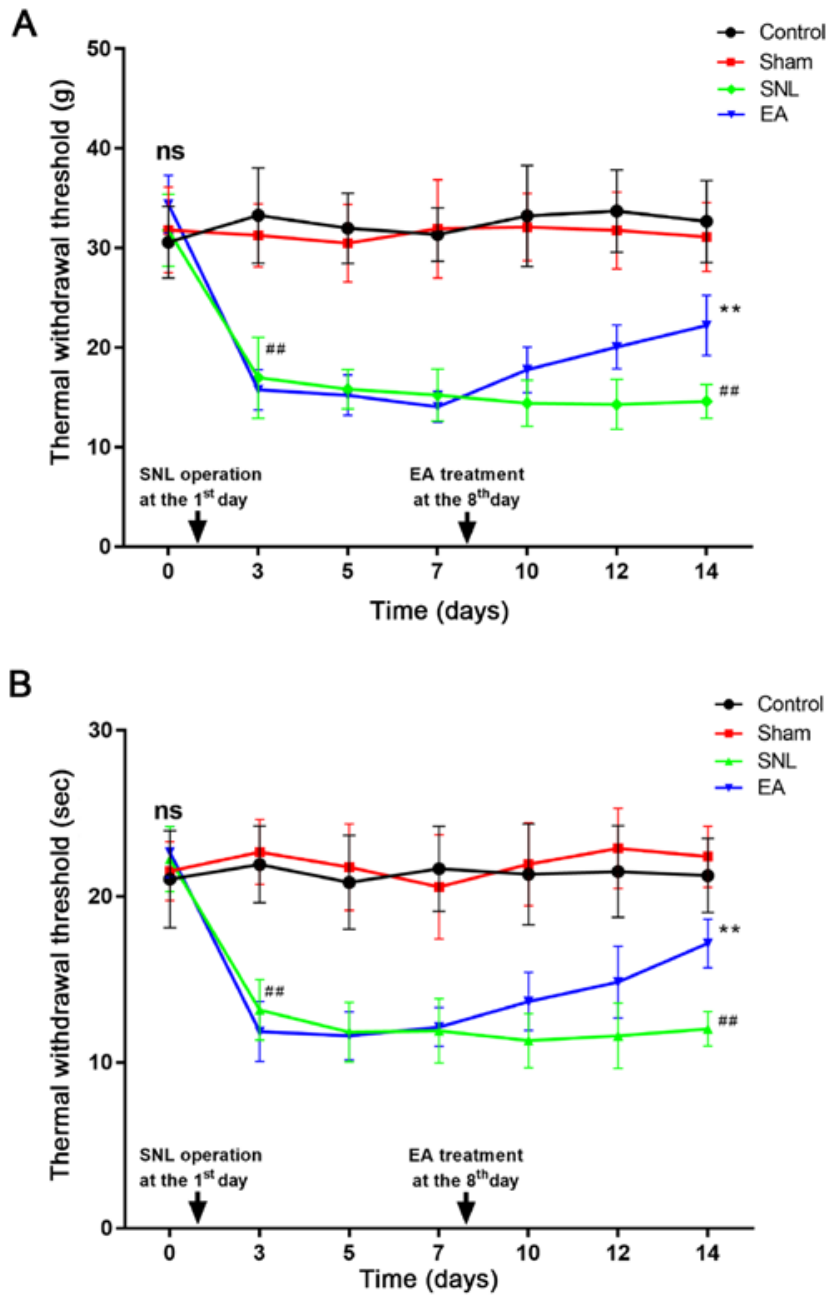

Figure 2. Treatment with EA results in a significant analgesic effect. (A) Mechanical allodynia. (B) Thermal hyperalgesia. The graph demonstrates the changes in withdrawal threshold. The threshold was observed pre- and post-EA. The values of MWT and TWL were notably decreased on day 3 post-SNL. The values of MWT and TWL of rats treated with EA were significantly higher than those in the SNL group. ${ }^{* *} \mathrm{P}<0.01$ vs. SNL group; ${ }^{\# \#} \mathrm{P}<0.01$ vs. sham group. EA, electroacupuncture; MWT, mechanical withdrawal threshold; TWL, thermal withdrawal latency; SNL, L-5 spinal nerve ligation; ns, not significant.

Patch-clamp recordings. The patch-clamp recording procedures were performed as previously described $(24,25)$. Specifically, the section was placed in a recording chamber beneath a BX51W1 upright light microscope (Olympus Corporation; magnification, x20). The procedure of tight-seal, whole-cell patch-clamp and the recordings were performed at room temperature $\left(22-24^{\circ} \mathrm{C}\right)$ with artificial cerebrospinal fluid perfusion. SG neurons were identified using an infrared and differential interference contrast camera (cat. no. BX51WI; Olympus Corporation; magnification, x20 and $\mathrm{x} 100)$. The recording pipettes were made from borosilicate glass capillaries (optical density, $1.5 \mathrm{~mm}$; inner diameter, $1.12 \mathrm{~mm}$; Sutter Instrument Company) with a micropipette puller (P-97; Sutter Instrument Company) and had a resistance of 4-6 M $\Omega$ when filled with a solution containing: $130.0 \mathrm{~K}$-gluconate, $5.0 \mathrm{KCl}, 4.0 \mathrm{Mg}$-ATP, 10.0 phosphocreatine, $0.3 \mathrm{Li}-\mathrm{GTP}$ and $10.0 \mathrm{mM}$ HEPES (pH 7.4 adjusted with $\mathrm{KOH}, 300 \mathrm{mOsm}$ ). The frequency of spontaneous excitatory postsynaptic currents (sEPSC) was recorded using an EPC-10 amplifier with a lowpass filter at $5 \mathrm{kHz}$ using Patchmaster software (version UI325; HEKA Elektronik GmbH; Harvard Bioscience, Inc.).
Statistical analysis. Statistical significance was determined using SPSS Statistics software (version 16.0; SPSS, Inc.). Data are presented as the mean \pm standard error of the mean of three experimental repeats. Behavioral results with multiple comparisons were statistically analyzed by a mixed analysis of variance (ANOVA) for repeated measures, followed by Sidak's test. The other data were carried out using one-way analysis of variance, followed by Tukey's test. $\mathrm{P}<0.05$ was considered to indicate a statistically significant difference.

\section{Results}

EA reverses SNL-induced mechanical allodynia and thermal hyperalgesia. Baseline measures of MWT and TWL did not differ between groups (Fig. 2). MWT and TWL were recorded on day 3 post-SNL to avoid measuring the effects of postoperative pain, as previously described (13). Mechanical allodynia and thermal allodynia developed at day 3 post-SNL and were sustained until day 14. In the EA groups, all rats were tested 30 min post-EA treatment. As presented in Fig. $2 \mathrm{~A}$ and B, the values of MWT and TWL in SNL rats notably decreased from day 3 to day 14 post-SNL compared with the control and sham 
A

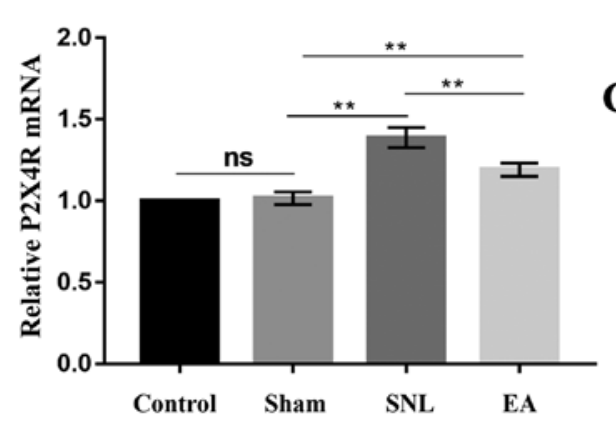

B

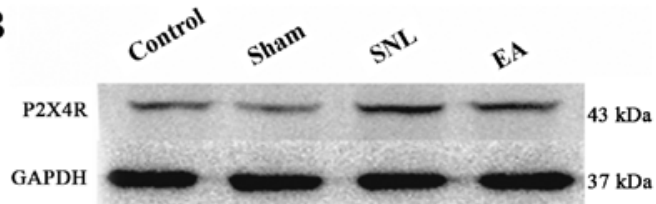

$\mathrm{C}$

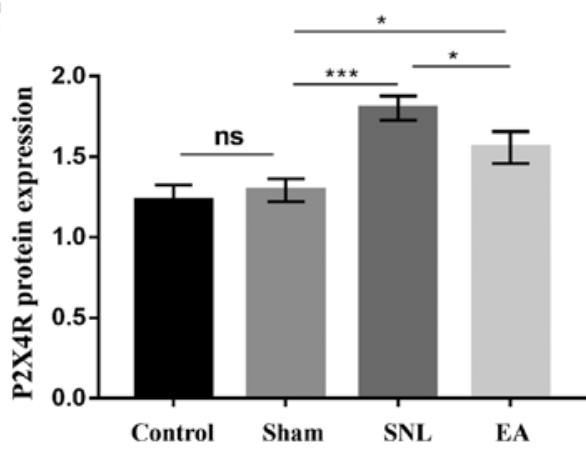

Figure 3. EA prevents P2X4R upregulation in SNL rats. (A) Quantification of relative P2X4R mRNA levels in SNL rats. (B) Western blotting analysis of total P2X4R protein in L4-L6 demonstrated notable upregulation in SNL rats. GAPDH was used as the loading control. (C) Summary data from western blotting experiments. ${ }^{*} \mathrm{P}<0.05 .{ }^{* *} \mathrm{P}<0.01 .{ }^{* * *} \mathrm{P}<0.001$. EA, electroacupuncture; $\mathrm{P} 2 \mathrm{X} 4 \mathrm{R}, \mathrm{P} 2 \mathrm{X} 4$ receptor; SNL, spinal nerve ligation; ns, not significant.
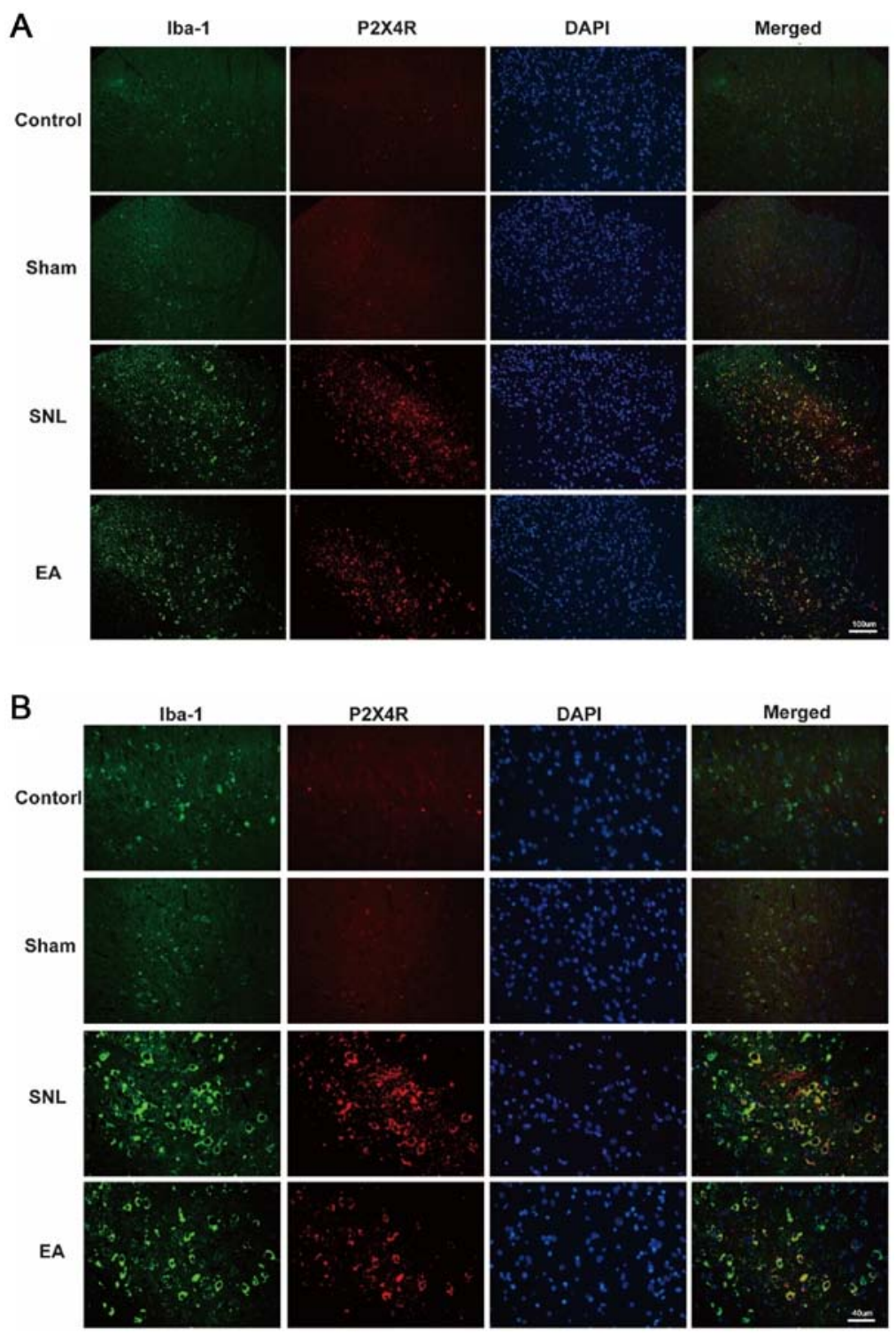

Figure 4. Representative photomicrographs of immunofluorescence of P2X4R (red) and Iba-1 (green) on the ipsilateral spinal dorsal horn on day 14 post-SNL (n=6). Scale bars, (A) 100 and (B) $40 \mu \mathrm{m}$. P2X4R, P2X4 receptor; SNL, spinal nerve ligation; Iba-1, ionized calcium-binding adapter molecule 1. 

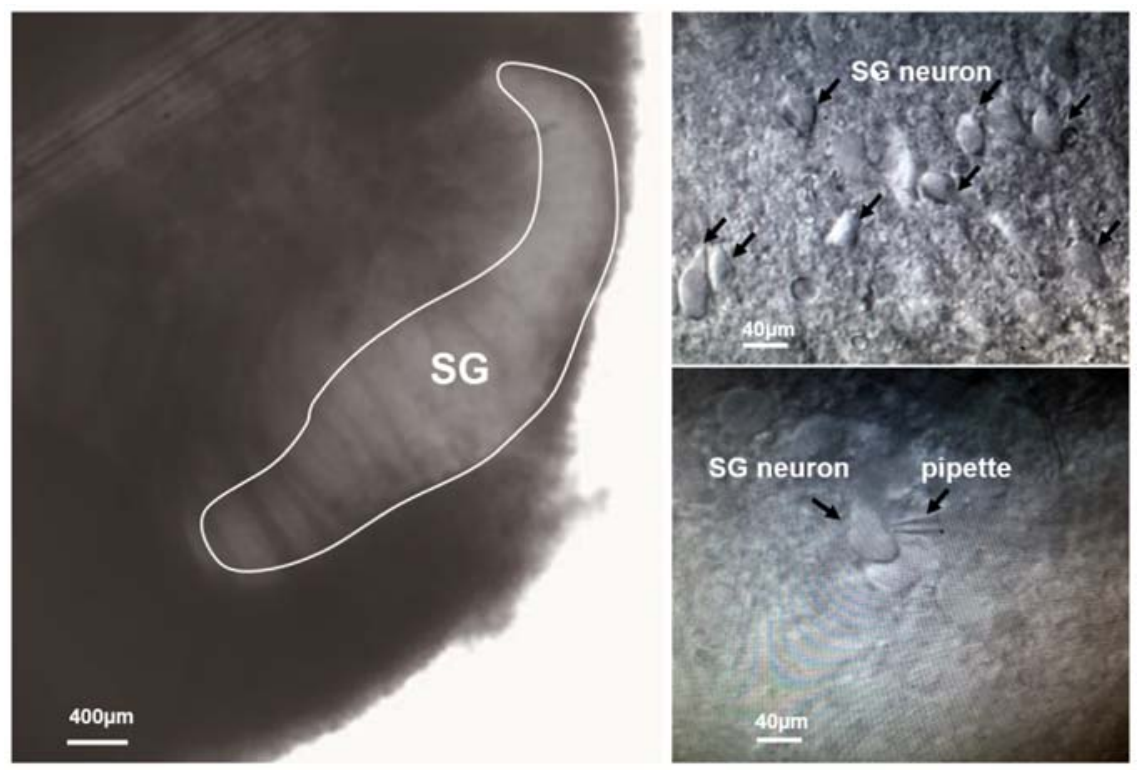

Figure 5. Representative images of the SG region in a transverse spinal cord section from a rat at low (left) and high magnification (right). SG, substantia gelatinosa.

A

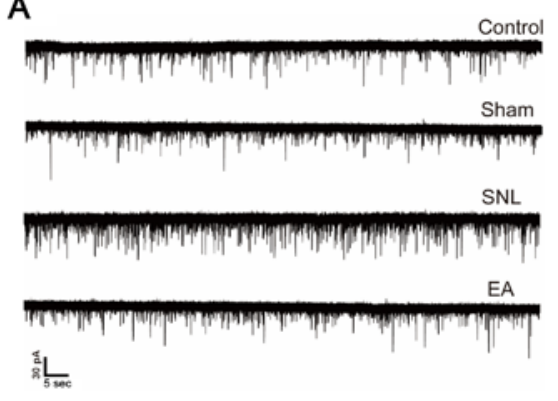

C

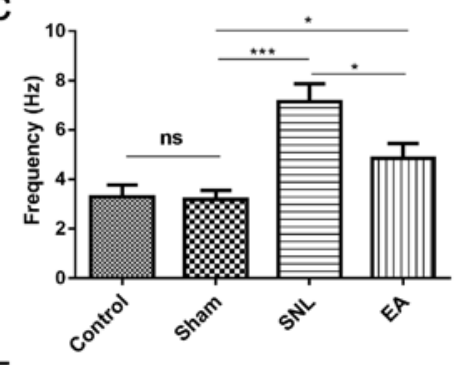

E

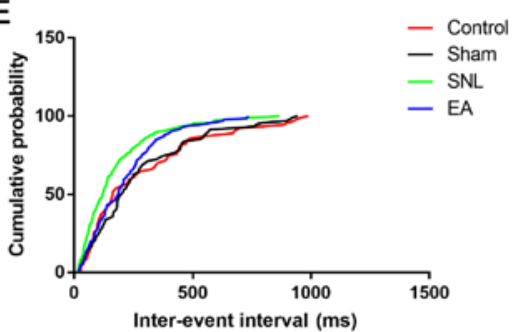

B

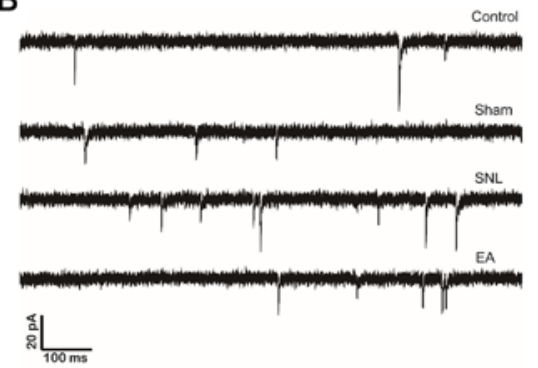

D

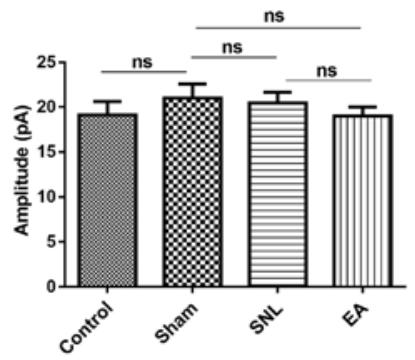

$\mathrm{F}$

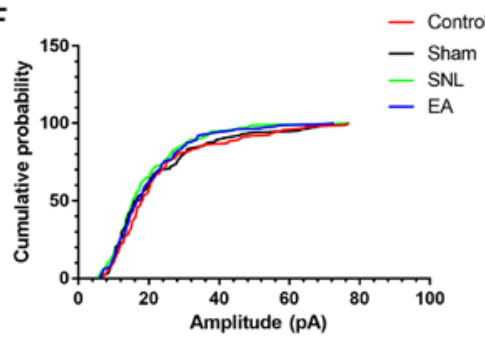

Figure 6. SNL dose-dependently increases the frequency but not the amplitude of sEPSCs in SG neurons. EA treatment decreased the frequency but not the amplitude of sEPSCs in SG neurons. (A) Representative recordings of sEPSCs obtained $2 \mathrm{~min}$ at a holding potential of $70 \mathrm{mV}$ after the current stabilized. (B) Enlarged view of representative recordings of sEPSCs. (C and D) Summary bar graphs of sEPSC frequency and amplitude in the four groups. SNL group exhibited an enhanced frequency of sEPSCs in SG neurons. EA treatment lowered the frequency of sEPSCs without altering the amplitude of sEPSCs. (E and F) Cumulative probability of the sEPSCs inter-event interval and amplitude plots from the same neuron. ${ }^{*} \mathrm{P}<0.05 .{ }^{* * * *} \mathrm{P}<0.001$. SNL, spinal nerve ligation; sEPSC, spontaneous excitatory postsynaptic currents; SG, substantia gelatinosa; EA, electroacupuncture; ns, not significant.

groups $(\mathrm{P}<0.01)$. In the EA groups, the values of MWT and TWL notably increased from day 7 to day 14 compared with those of the SNL groups $(\mathrm{P}<0.01)$. The results indicate that EA relieved pain behavior in SNL rats. 
EA decreases expression levels of P2X4R $m R N A$ and protein in the spinal cord of $S N L$ rats. In order to detect the effect of EA and to demonstrate the role of P2X4R in maintaining $\mathrm{NP}$, the mRNA and protein levels of P2X4R in the spinal cord were investigated. As presented in Fig. 3A, P2X4R mRNA expression levels in the SNL group were higher than those in the control and sham groups $(\mathrm{P}<0.01)$. However, the relative expression levels of $\mathrm{P} 2 \mathrm{X} 4 \mathrm{R}$ mRNA in the EA group decreased in comparison with those in the SNL group $(\mathrm{P}<0.01)$ following 7 days of EA treatment. The results of $\mathrm{P} 2 \mathrm{X} 4 \mathrm{R}$ protein expression level analysis are presented in Fig. 3B and C. The relative expression levels of P2X4R protein in the control, sham, SNL and EA groups were $1.229 \pm 0.043,1.291 \pm 0.031,1.800 \pm 0.034$ and $1.557 \pm 0.044$, respectively. The SNL group exhibited upregulated $\mathrm{P} 2 \mathrm{X} 4 \mathrm{R}$ protein levels compared with those in the control and sham groups $(\mathrm{P}<0.001)$. The $\mathrm{P} 2 \mathrm{X} 4 \mathrm{R}$ protein levels in the EA group were significantly lower than those in the SNL group $(\mathrm{P}<0.05)$. The results indicate that EA inhibited the upregulation of $\mathrm{P} 2 \mathrm{X} 4 \mathrm{R}$ protein expression levels in SNL rats.

EA decreases immunofluorescence staining of $P 2 X 4 R$ and $I b a-1$. Upregulation of ionized calcium-binding adapter molecule 1 (Iba-1) is a marker of microglia activation (26). The results of double immunofluorescence staining are presented in Fig. 4A (magnification, x20) B (magnification, $\mathrm{x} 40$ ). The co-expression of P2X4R and Iba-1 was notable in the SNL group in contrast with the control group. The number of $\mathrm{P} 2 \mathrm{X} 4 \mathrm{R}^{+}$microglia in the EA group was significantly lower following EA treatment. These results indicate that EA inhibited microglia activation and suppressed the expression levels of the P2X4R receptor.

EA decreases the frequency of sEPSCs in spinal cord $S G$ neurons in the SNL group. Spinal cord SG neurons are predominantly excitatory neurons (24) and form a nociceptive circuit, which receives input from afferent $\mathrm{C}$-fibers and sends output to lamina I projection neurons (27). It was hypothesized that EA may modulate neurotransmitter release and synaptic transmission by increasing expression levels of $\mathrm{P} 2 \mathrm{X} 4 \mathrm{R}$ in the spinal cord. The present study recorded SEPSCs in SG neurons in spinal cord sections from rats (Figs. 5 and 6A and B). The SNL group exhibited an enhanced frequency of sEPSCs in SG neurons $(\mathrm{P}<0.001$; Fig. $6 \mathrm{C}$ and $\mathrm{E})$ compared with the control and sham groups. In the EA group the frequency of sEPSCs was significantly decreased $(\mathrm{P}<0.05$; Fig. $6 \mathrm{C}$ and $\mathrm{E})$ but the amplitude of sEPSCs was not significantly altered $(\mathrm{P}>0.05$; Fig. 6D and F) compared with the SNL group.

Glutamate AMPA/kainate receptors mediate sEPSCs, and excitatory synaptic transmission causes frequency changes in sEPSCs (28-30). Therefore, EA may inhibit excitatory synaptic transmission by decreasing glutamate release from presynaptic terminals, which may result in EA-induced suppression of P2X4R expression levels in microglia.

\section{Discussion}

The results of the present study demonstrated that EA treatment alleviated nerve injury-induced tactile allodynia and thermal hyperalgesia by inhibiting activation of spinal microglia-mediated P2X4R and by regulating the excitability of neurons in the SG region of SNL rats. The findings of the present study demonstrated the underlying mechanisms of the therapeutic effect of EA on NP in regards to purinergic receptor family modulation.

Acupuncture is used worldwide as a treatment for a number of conditions, particularly for pain $(4,31)$. Zusanli and Kunlun points, first described in 'HuangDiNeiJing-LingShu-BenShu' (an ancient Chinese book, recorded in 200 BC), are commonly used acupoints to treat a number of symptoms (including pain relief) both in clinical practice and in research. It has been reported that EA stimulates the Zusanli point to relieve NP via inhibition of COX2 expression levels, activation of opioid receptors $\mathrm{M} 1 \mathrm{mAChR}, \beta 2 \mathrm{nAChR}$ and endothelin-B receptors, and secretion of neuroactive mediators (32-34). Studies have also demonstrated that acupuncture at the Kunlun point can alleviate NP by inhibiting the p38 MAPK pathway and the expression levels of prostaglandin E2 and G protein-coupled kinase 2 (35,36). In the present study, increased sensitivity to thermal and mechanical stimulation was observed in SNL rats. SNL rats exhibit abnormal hyperalgesia and mechanical irritation, which is similar to human NP symptoms and behavior induced by injury and dysfunction of the peripheral nervous system (37). In the present study, the MWT and TWL in the EA group were significantly increased compared with the SNL group, indicating that EA may relieve mechanical pain and thermal pain in SNL rats. These findings indicated that EA treatment at the Zusanli and Kunlun points may be beneficial in the treatment of NP.

Spinal microglia have been demonstrated to be immediately activated following nerve injury and are necessary for the initiation and maintenance of pain hypersensitivity (38). The ATP receptors P2X4R and P2X7R have been demonstrated to be predominately expressed in the microglia of the spinal cord $(11,39,40)$. Furthermore, a previous study has demonstrated that DRG P2X3R is involved in the analgesic effect of EA in rat models of chronic constriction injury (13). Following binding of ATP, microglial ionotropic P2X4R leads to increased microglia activation, which exaggerates pain states (41). Inhibition of spinal $\mathrm{P} 2 \mathrm{X} 4 \mathrm{R}^{+}$microglia significantly alleviates tactile allodynia induced by nerve injury but not that induced by thermal hyperalgesia $(11,42)$. Moreover, P2X4R knockout has been demonstrated to increase sensitivity to thermal hyperalgesia in an inflammatory mouse model (43). In the present study, the expression levels of P2X4R protein and mRNA were notably decreased compared with those in SNL rats following EA treatment. Therefore, the effects of EA on NP may be associated with the expression levels of P2X4R. Moreover, the results of the present study indicate that P2X4R was co-expressed with Iba-1 in the SDH. These results are consistent with the results of previous studies $(11,44)$. The present study also demonstrated that co-expression of $\mathrm{P} 2 \mathrm{X} 4 \mathrm{R}$ and Iba-1 in the SDH of SNL rats was increased compared with that in control rats. Upregulated Iba-1 was associated with the activation of microglia. The results of the present study indicated that microglia were activated following PNI. Following EA treatment, the co-expression of P2X4R with Iba-1 in the SDH was decreased compared with that in the SNL group. EA may attenuate the transmission of nociceptive information by inhibiting the expression levels of P2X4R in SDH microglia, thus relieving pain behaviors in SNL rats. 
sEPSCs were the most important indicator reflecting the excitatory transmission of neurons recorded. In general, the frequency of sEPSC changes was associated with presynaptic mechanisms. Increased presynaptic transmitter release led to increased sEPSC frequency. The amplitude of sEPSC was associated with pre- and post-synaptic mechanisms. In the present study, whole-cell patch clamp results demonstrated no significant difference in the amplitude of sEPSCs in spinal SG neurons in the four groups, but the frequency of sEPSCs was significantly different. Compared with the control group, the SNL group exhibited higher sEPSC frequency in SG nociceptive neurons. Additionally, the present study demonstrated that EA significantly decreased the frequency of sEPSCs but did not affect the amplitude of sEPSCs. These results indicated that EA may attenuate the transmission efficiency between synapses in the SG region of the SDH during NP by decreasing the excitability of neurons that transmit pain signals from the peripheral nerves to the spinal cord. In addition, previous studies have also demonstrated that EA has anti-inflammatory effects (45-48) and that microglia activation is associated with inflammation (49). The SNL rat model can induce both NP and inflammatory pain (50-52). The present study indicates that the mechanism underlying EA treatment of NP involves the transmission efficiency between synapses.

In light of the downregulated P2X4R mRNA and protein expression levels in the EA group, the present study demonstrated that the analgesic effects of EA analgesia may be mediated in part by P2X4R. In future, pharmacological, chemogenic and optogenetic methods may be used to further characterize the analgesic effects of EA mediated by P2X4R.

In conclusion, the present study demonstrated that mechanical allodynia and thermal hyperalgesia may be attenuated by the analgesic effects of EA. EA may exert analgesic effects by inhibiting P2X4R-mediated activation of spinal microglia and decreasing the excitability of neurons in the SG region of SNL rats. However, further research is required in order to verify these effects and to identify the underlying molecular mechanism of EA in animals with PNI.

\section{Acknowledgements}

The authors would like to thank Dr Yu Su and Dr Lixiu Lv in the Scientific Research Center of The Second Affiliated Hospital and Yuying Children's Hospital of Wenzhou Medical University, Wenzhou, China.

\section{Funding}

The current study was supported by National Natural Science Foundation of China (grant nos. 81574074 and 81873376) and the Basic Research Program of Wenzhou City (grant no. Y20190192).

\section{Availability of data and materials}

The datasets generated during and/or analyzed during the current study are available from the corresponding author on reasonable request.

\section{Authors' contributions}

YZ, CJ, XJ, JC, XC and XY performed the laboratory experiments, collected and analyzed the data and interpreted the results. $\mathrm{KZ}$ wrote the manuscript. JW, MJ and GY analyzed the data. MJ and GY revised the manuscript. KZ, WT and SJ designed the experiments, supervised the study and revised the manuscript. All authors read and approved the final manuscript.

\section{Ethics approval and consent to participate}

All applicable international, national, and/or institutional guidelines for the care and use of animals were followed. All experiments were approved by the Institutional Animal Care and Use Committee of Wenzhou Medical University (approval no. WMU 174890).

\section{Patient consent for publication}

Not applicable.

\section{Competing interests}

The authors declare that they have no competing interests.

\section{References}

1. Wang JY, Chen R, Chen SP, Gao YH, Zhang JL, Feng XM, Yan Y, Liu JL, Gaischek I, Litscher D, et al: Electroacupuncture reduces the effects of acute noxious stimulation on the electrical activity of pain-related neurons in the hippocampus of control and neuropathic pain rats. Neural Plast 2016: 6521026, 2016.

2. Wang Y, Zhao Y, Ma X, Li J, Hou J and Lv X: Beneficial Effects of Electroacupuncture on Neuropathic Pain Evoked by Spinal Cord Injury and Involvement of PI3K mTOR Mechanisms. Biol Res Nurs 21: 5-13, 2019.

3. Chen XM, Xu J, Song JG, Zheng BJ and Wang XR: Electroacupuncture inhibits excessive interferon- $\gamma$ evoked up-regulation of P2X4 receptor in spinal microglia in a CCI rat model for neuropathic pain. Br J Anaesth 114: 150-157, 2015.

4. Zhao ZQ: Neural mechanism underlying acupuncture analgesia. Prog Neurobiol 85: 355-375, 2008.

5. Park JH, Kim SK, Kim HN, Sun B, Koo S, Choi SM, Bae H and Min BI: Spinal cholinergic mechanism of the relieving effects of electroacupuncture on cold and warm allodynia in a rat model of neuropathic pain. J Physiol Sci 59: 291-298, 2009.

6. Kim SK, Park JH, Bae SJ, Kim JH, Hwang BG, Min BI, Park DS and Na HS: Effects of electroacupuncture on cold allodynia in a rat model of neuropathic pain: Mediation by spinal adrenergic and serotonergic receptors. Exp Neurol 195: 430-436, 2005.

7. Padi SS and Kulkarni SK: Minocycline prevents the development of neuropathic pain, but not acute pain: Possible anti-inflammatory and antioxidant mechanisms. Eur J Pharmacol 601: 79-87, 2008.

8. Tsuda M, Tozaki-Saitoh H and Inoue K: Purinergic system, microglia and neuropathic pain. Curr Opin Pharmacol 12: 74-79, 2012.

9. Beggs S, Trang T and Salter MW: P2X4R ${ }^{+}$microglia drive neuropathic pain. Nat Neurosci 15: 1068-1073, 2012.

10. Sun S, Cao H, Han M, Li TT, Zhao ZQ and Zhang YQ: Evidence for suppression of electroacupuncture on spinal glial activation and behavioral hypersensitivity in a rat model of monoarthritis. Brain Res Bull 75: 83-93, 2008.

11. Tsuda M, Shigemoto-Mogami Y, Koizumi S, Mizokoshi A, Kohsaka S, Salter MW and Inoue K: P2X4 receptors induced in spinal microglia gate tactile allodynia after nerve injury. Nature 424: 778-783, 2003.

12. Tsuda M and Inoue K: Neuron microglia interaction by purinergic signaling in neuropathic pain following neurodegeneration. Neuropharmacology, 2015. 
13. Tu WZ, Cheng RD, Cheng B, Lu J, Cao F, Lin HY, Jiang YX, Wang JZ, Chen $\mathrm{H}$ and Jiang SH: Analgesic effect of electroacupuncture on chronic neuropathic pain mediated by $\mathrm{P} 2 \mathrm{X} 3$ receptors in rat dorsal root ganglion neurons. Neurochem Int 60: 379-386, 2012

14. Xu J, Chen XM, Zheng BJ and Wang XR: Electroacupuncture relieves nerve injury-induced pain hypersensitivity via the inhibition of spinal P2X7 receptor-positive microglia. Anesth Analg 122: 882-892, 2016

15. Grace PM, Rolan PE and Hutchinson MR: Peripheral immune contributions to the maintenance of central glial activation underlying neuropathic pain. Brain Behav Immun 25: 1322-1332, 2011.

16. Tsuda M, Masuda T, Kitano J, Shimoyama H, Tozaki-Saitoh $H$ and Inoue $\mathrm{K}$ : IFN- $\gamma$ receptor signaling mediates spinal microglia activation driving neuropathic pain. Proc Natl Acad Sci USA 106: 8032-8037, 2009 .

17. Phelps CE, Navratilova E, Dickenson AH, Porreca F and Bannister K: Kappa opioid signaling in the right central amygdala causes hind paw specific loss of diffuse noxious inhibitory controls in experimental neuropathic pain. Pain 160: $1614-1621,2019$.

18. Navratilova E, Ji G, Phelps C, Qu C, Hein M, Yakhnitsa V, Neugebauer V and Porreca F: Kappa opioid signaling in the central nucleus of the amygdala promotes disinhibition and aversiveness of chronic neuropathic pain. Pain 160: 824-832, 2019.

19. Sosanya NM, Kumar R, Clifford JL, Chavez R, Dimitrov G Srinivasan S, Gautam A, Trevino AV, Williams M, Hammamieh R, et al: Identifying Plasma Derived Extracellular Vesicle (EV) Contained Biomarkers in the Development of Chronic Neuropathic Pain. J Pain: Jun 19, 2019 (Epub ahead of print).

20. Gofman L, Fernandes NC and Potula R: Relative Role of Akt, ERK and CREB in Alcohol-Induced Microglia P2X4R Receptor Expression. Alcohol Alcohol 51: 647-654, 2016.

21. Livak KJ and Schmittgen TD: Analysis of relative gene expression data using real-time quantitative PCR and the 2(-Delta Delta C(T)) Method. Methods 25: 402-408, 2001.

22. Zhou K, Wu J, Chen J, Zhou Y, Chen X, Wu Q, Xu Y, Tu W, Lou X, Yang G, et al: Schaftoside ameliorates oxygen glucose deprivation-induced inflammation associated with the TLR4/Myd88/Drp1-related mitochondrial fission in BV2 microglia cells. J Pharmacol Sci 139: 15-22, 2019.

23. Zhou K, Chen J, Wu J, Wu Q, Jia C, Xu YXZ, Chen L, Tu W Yang G, Kong J, et al: Atractylenolide III ameliorates cerebral ischemic injury and neuroinflammation associated with inhibiting JAK2/STAT3/Drp1-dependent mitochondrial fission in microglia. Phytomedicine 59: 152922, 2019.

24. Rivera-Arconada I, Roza C and Lopez-Garcia JA: Characterization of hyperpolarization-activated currents in deep dorsal horn neurons of neonate mouse spinal cord in vitro. Neuropharmacology 70: 148-155, 2013.

25. Yasaka T, Tiong SY, Hughes DI, Riddell JS and Todd AJ: Populations of inhibitory and excitatory interneurons in lamina II of the adult rat spinal dorsal horn revealed by a combined electrophysiological and anatomical approach. Pain 151: 475-488, 2010.

26. Sankar SB, Pybus AF, Liew A, Sanders B, Shah KJ, Wood LB and Buckley EM: Low cerebral blood flow is a non-invasive biomarker of neuroinflammation after repetitive mild traumatic brain injury. Neurobiol Dis 124: 544-554, 2019.

27. Todd AJ: Neuronal circuitry for pain processing in the dorsal horn. Nat Rev Neurosci 11: 823-836, 2010.

28. Kohno T, Wang H, Amaya F, Brenner GJ, Cheng JK, Ji RR and Woolf CJ: Bradykinin enhances AMPA and NMDA receptor activity in spinal cord dorsal horn neurons by activating multiple kinases to produce pain hypersensitivity. J Neurosci 28: 4533-4540, 2008.

29. Yang K, Kumamoto E, Furue $\mathrm{H}$ and Yoshimura M: Capsaicin facilitates excitatory but not inhibitory synaptic transmission in substantia gelatinosa of the rat spinal cord. Neurosci Lett 255: 135-138, 1998.

30. Kawasaki Y, Zhang L, Cheng JK and Ji RR: Cytokine mechanisms of central sensitization: Distinct and overlapping role of interleukin- $1 \beta$, interleukin- 6 , and tumor necrosis factor- $\alpha$ in regulating synaptic and neuronal activity in the superficial spinal cord. J Neurosci 28: 5189-5194, 2008.

31. Berman BM,Langevin HM, Witt CM and Dubner R: Acupuncture for chronic low back pain. N Engl J Med 363: 454-461, 2010.

32. Lau WK, Lau YM, Zhang HQ, Wong SC and Bian ZX: Electroacupuncture versus celecoxib for neuropathic pain in rat SNL model. Neuroscience 170: 655-661, 2010.
33. Chen SP, Kan Y, Zhang JL, Wang JY, Gao YH, Qiao LN, Feng XM, Yan YX and Liu JL: Involvement of hippocampal acetylcholinergic receptors in electroacupuncture analgesia in neuropathic pain rats. Behav Brain Funct 12: 13, 2016.

34. Vieira JS, Toreti JA, de Carvalho RC, de Araújo JE, Silva ML and Silva JRT: Analgesic Effects Elicited by Neuroactive Mediators Injected into the ST 36 Acupuncture Point on Inflammatory and Neuropathic Pain in Mice. J Acupunct Meridian Stud 11: 280-289, 2018.

35. Liang Y, Du JY, Qiu YJ, Fang JF, Liu J and Fang JQ: Electroacupuncture attenuates spinal nerve ligation-induced microglial activation mediated by p38 mitogen-activated protein kinase. Chin J Integr Med 22: 704-713, 2016.

36. Jiang H, Yu X, Ren X and Tu Y: Electroacupuncture alters pain related behaviors and expression of spinal prostaglandin E2 in a rat model of neuropathic pain. J Tradit Chin Med 36: 85-91, 2016.

37. Gao Y, Xu C, Liang S, Zhang A, Mu S, Wang Y and Wan F: Effect of tetramethylpyrazine on primary afferent transmission mediated by $\mathrm{P} 2 \mathrm{X} 3$ receptor in neuropathic pain states. Brain Res Bull 77: 27-32, 2008

38. Inoue $\mathrm{K}$ and Tsuda $\mathrm{M}$ : Microglia and neuropathic pain. Glia 57: $1469-1479,2009$

39. He WJ, Cui J, Du L, Zhao YD, Burnstock G, Zhou HD and Ruan HZ: Spinal P2X(7) receptor mediates microglia activation-induced neuropathic pain in the sciatic nerve injury rat model. Behav Brain Res 226: 163-170, 2012.

40. Kobayashi K, Takahashi E, Miyagawa Y, Yamanaka H and Noguchi K: Induction of the P2X7 receptor in spinal microglia in a neuropathic pain model. Neurosci Lett 504: 57-61, 2011.

41. Masuda T, Iwamoto S, Yoshinaga R, Tozaki-Saitoh $\mathrm{H}$, Nishiyama A, Mak TW, Tamura T, Tsuda M and Inoue K: Transcription factor IRF5 drives $\mathrm{P} 2 \mathrm{X} 4 \mathrm{R}^{+}$-reactive microglia gating neuropathic pain. Nat Commun 5: 3771, 2014

42. Biber K, Tsuda M, Tozaki-Saitoh H, Tsukamoto K, Toyomitsu E, Masuda T, Boddeke H and Inoue K: Neuronal CCL21 up-regulates microglia P2X4 expression and initiates neuropathic pain development. EMBO J 30: 1864-1873, 2011

43. Ulmann L, Hirbec H and Rassendren F: P2X4 receptors mediate PGE2 release by tissue-resident macrophages and initiate inflammatory pain. EMBO J 29: 2290-2300, 2010.

44. Williams WA, Linley JE, Jones CA, Shibata Y, Snijder A, Button J, Hatcher JP, Huang L, Taddese B, Thornton P, et al: Jones Antibodies binding the head domain of P2X4 inhibit channel function and reverse neuropathic pain. Pain 160: 1989-2003, 2019.

45. Gao F, Xiang HC, Li HP, Jia M, Pan XL, Pan HL and Li M: Electroacupuncture inhibits NLRP3 inflammasome activation through CB2 receptors in inflammatory pain. Brain Behav Immun 67: 91-100, 2017.

46. Zhan J, Qin W, Zhang Y, Jiang J, Ma H, Li Q and Luo Y: Upregulation of neuronal zinc finger protein A20 expression is required for electroacupuncture to attenuate the cerebral inflammatory injury mediated by the nuclear factor-kB signaling pathway in cerebral ischemia/reperfusion rats. J Neuroinflammation 13: 258, 2016.

47. Zhang R, Lao L, Ren K and Berman BM: Mechanisms of acupuncture-electroacupuncture on persistent pain. Anesthesiology 120: 482-503, 2014.

48. Zhang Y, Zhang RX, Zhang M, Shen XY, Li A, Xin J, Ren K, Berman BM, Tan M and Lao L: Electroacupuncture inhibition of hyperalgesia in an inflammatory pain rat model: Involvement of distinct spinal serotonin and norepinephrine receptor subtypes. Br J Anaesth 109: 245-252, 2012.

49. Harrison C: Inflammatory disorders: Steroids modulate microglia-mediated inflammation. Nat Rev Drug Discov 10: 492-493, 2011.

50. Zhuang ZY, Gerner P, Woolf CJ and Ji RR: ERK is sequentially activated in neurons, microglia, and astrocytes by spinal nerve ligation and contributes to mechanical allodynia in this neuropathic pain model. Pain 114: 149-159, 2005.

51. Liu X, Liu H, Xu S, Tang Z, Xia W, Cheng Z, Li W and Jin Y: Spinal translocator protein alleviates chronic neuropathic pain behavior and modulates spinal astrocyte-neuronal function in rats with L5 spinal nerve ligation model. Pain 157: 103-116, 2016

52. Burke NN, Kerr DM, Moriarty O, Finn DP and Roche M: Minocycline modulates neuropathic pain behaviour and cortical M1-M2 microglial gene expression in a rat model of depression. Brain Behav Immun 42: 147-156, 2014.

This work is licensed under a Creative Commons Attribution-NonCommercial-NoDerivatives 4.0 International (CC BY-NC-ND 4.0) License. 